\title{
Risk perception of NSAIDs in South Dakota in comparison with Slovakia and Greece
}

\author{
Varga $\mathrm{Z}^{1}$, Haiar $\mathrm{JM}^{1}$, Oberoi $\mathrm{M}^{1}$, Thorp $\mathrm{A}^{1}$, Petrasko $\mathrm{P}^{1}$, Kremer $\mathrm{A}^{1}$, Kristova $\mathrm{V}^{2}$, Kriska $\mathrm{M}^{2}$, \\ Stys $\mathrm{T}^{1}$, Stys $\mathrm{A}^{1}$
}

Sanford Heart Hospital, Sioux Falls, South Dakota, USA, University of South Dakota Sanford School of Medicine. zoltan.varga.md.phd@gmail.com

\begin{abstract}
AIM: Adverse effects (ADRs) of non-steroidal anti-inflammatory drugs (NSAIDs) represent a public health problem. To decrease the negative effect on the population, an improvement of risk awareness is crucial. We aimed to evaluate the risk perception and the use of NSAIDs in South Dakota in comparison with Slovakia and Greece.

METHOD: A structured questionnaire evaluating NSAID use in 185 patients in a hospital in South Dakota. RESULTS: $95.7 \%$ of respondents reported the use of analgesics. On 1-10 visual analogue scale, perceived risk of NSAIDs was $4.27 \pm 2.46$, similar to Greece $(4.36 \pm 2.41, p=0.360)$, but significantly higher than in Slovakia $(3.8 \pm 1.9, p=0.038)$. Only $12.4 \%$ were familiar with gastrointestinal ADRs and only $1.1 \%$ were aware of cardiovascular risk. Although $57.8 \%$ were informed about ADRs by their doctor or pharmacist, only 33.0 $\%$ were informed spontaneously, without actively asking. Providers in South Dakota were informing patients spontaneously more often than in Slovakia $(15.9 \%, p \leq 0.001)$ and on par with Greece $(36.3 \%, p=0.631)$. CONCLUSIONS: Public awareness about NSAID risk is dangerously low. Only a third of providers are informing patients about possible risks spontaneously (Tab. 6, Ref. 15). Text in PDF www.elis.sk KEY WORDS: non-steroidal anti-inflammatory drugs, risk perception, adverse effects, cardiovascular risk, gastrointestinal risk.
\end{abstract}

\section{Introduction}

Non-steroidal anti-inflammatory drugs (NSAIDs) belong to the most used and prescribed pharmacologic agents worldwide. At the same time, they put patients at risk of developing potentially serious, even life-threatening adverse drug reactions (ADR) (1). The sheer number of people exposed to drugs from the group of NSAIDs, and the fact that the majority of them are available overthe-counter (OCT), means that their ADRs represent a significant public health problem (2).

NSAID use might lead to formation of ulcers in the gastrointestinal tract (3), thrombotic events including myocardial infarction $(\mathrm{MI})(4,5)$, decreased kidney function $(6,7)$, stroke $(8$, $9)$, increased systemic arterial blood pressure (BP) $(10,11)$ or exacerbation of congestive heart failure (CHF) (5). Potentially dangerous interactions with other frequently used medications are also common (12).

${ }^{1}$ Sanford Cardiovascular Institute, Sanford Heart Hospital, Sioux Falls, South Dakota, USA, University of South Dakota Sanford School of Medicine, Sioux Falls, SD, USA, and ${ }^{2}$ Institute of Pharmacology and Clinical Pharmacology, Faculty of Medicine, Comenius University in Bratislava, Bratislava, Slovakia

Address for correspondence: $\mathrm{Z}$. Varga, MD, PhD, Sanford Cardiovascular Institute, Sanford Heart Hospital, Sioux Falls, South Dakota, USA, University of South Dakota Sanford School of Medicine, 1301 W. $18^{\text {th }}$ St., Sioux Falls, SD, 57 105, USA
To decrease the negative effect of ADRs of NSAIDs on the population, a continuous effort is required to analyse NSAID use in the population and evaluate the risk of ADRs. Improvements in user awareness of NSAID mediated risks are also crucial.

In the study presented below, we used a questionnaire to evaluate the use of NSAIDs in patients hospitalized due to internal medicine primary diagnosis in a university hospital setting in South Dakota. The questionnaire was also used to gather information about NSAID-related knowledge and the level of risk perception. We assessed the presence of selected factors/comorbid conditions that could potentially raise the risk of cardiovascular (CV) adverse effects of NSAIDs. Eventually, the results were compared to our prior results obtained using similar questionnaires in two European Union countries, Slovakia and Greece.

\section{Materials and methods}

The questionnaire used to conduct the study contained 18 questions ( 6 closed ended, 9 semi-open ended, 1 open ended and 2 visual analogue scales). The questionnaire was administered by one of the authors face-to-face in the inpatient setting. In case the patient needed clarification about any of the questions, clarification was promptly provided.

The study group consisted of 185 patients, who were randomly selected from the pool of patients hospitalized within the internal medicine service in the participating university hospital located 
in South Dakota from July of 2019 to November of 2019. Patients listed under a non-internal medicine or those, who were admitted with non-internal medicine primary diagnosis were not allowed to participate. Other exclusion criteria were inability to complete the questionnaire for reasons of advanced dementia, altered mentation, speech/language barrier or unwillingness to participate. Our response rate was over $95 \%$.

Our data was evaluated with the methods of descriptive statistics. Comparisons between the results of the presented study and our prior results from the study populations in Slovakia and Greece were done with the use of ANOVA, t-test and chi-squared test. $P$ value under 0.05 was considered statistically significant. Results are shown as the average \pm standard deviation (SD).

The presented study was conducted in accordance with all relevant laws of the United States of America and the state of South Dakota.

\section{Results}

A total number of 185 inpatient respondents were able to fill out the questionnaire. The gender distribution was balanced, with

Tab. 1. NSAIDs and other non-opioid analgesics used by patients.

\begin{tabular}{lcc}
\hline Drug & Number of patients & Percentage \\
\hline Paracetamol (acetaminophen) & 137 & $74.1 \%$ \\
Ibuprofen $_{\text {Naproxen }}$ & 88 & $47.6 \%$ \\
Acetylsalicylic acid (Aspirin) & 57 & $30.8 \%$ \\
Other & 33 & $17.8 \%$ \\
\hline
\end{tabular}

analgesic-antipyretic

Tab. 2. Specific regions of the body where pain prompts patients to take pain medications.

\begin{tabular}{lcc}
\hline Region & Number of patients & Percentage \\
\hline Head & 93 & $50.3 \%$ \\
Back & 81 & $43.8 \%$ \\
Arms/legs & 63 & $34.1 \%$ \\
Tooth & 26 & $14.1 \%$ \\
Stomach & 24 & $13.0 \%$ \\
Chest & 15 & $8.1 \%$ \\
Other & 30 & $16.2 \%$ \\
\hline
\end{tabular}

Tab. 3. Sources of information for learning about the risks of taking pain medications.

\begin{tabular}{lcc}
\hline Source & Number of patients & Percentage \\
\hline Documentation which came with the pain medication & 54 & $29.2 \%$ \\
Doctor & 45 & $24.3 \%$ \\
Pharmacist & 33 & $17.8 \%$ \\
Internet search & 23 & $12.4 \%$ \\
Other patients & 7 & $3.8 \%$ \\
Never tried to find such information & 92 & $49.7 \%$ \\
\hline
\end{tabular}

Tab. 4. Percentage of patients reporting being informed about ADRs by a healthcare professional.

\begin{tabular}{|c|c|c|c|}
\hline & South Dakota $(n=185)$ & Slovakia $(n=251)$ & Greece $(n=124)$ \\
\hline Percentage of patients reporting being informed about ADRs & $57.8 \%$ & $31.1 \%$ & $49.2 \%$ \\
\hline Percentage reporting being informed spontaneously & $33.0 \%$ & $15.9 \%$ & $36.3 \%$ \\
\hline
\end{tabular}

$\mathrm{ADR}$ - adverse drug reaction
$53.9 \%(n=97)$ of respondents being female and $46.1 \%(n=83)$ male. The average age of our respondents was $63.6 \pm 17.5$ years. The overwhelming majority $(\mathrm{n}=177,95.7 \%)$ reported at least an occasional use of analgesics. Nearly half of the respondents $(\mathrm{n}=$ $80,45.2 \%$ ) reported at least weekly to monthly use. Paracetamol (acetaminophen) was the most commonly used analgesic $(\mathrm{n}=137$, $74.1 \%$ of the study group), followed by ibuprofen ( $\mathrm{n}=88,47.6 \%)$ and naproxen $(\mathrm{n}=57,30.8 \%$ ) (Tab. 1). None of our respondents reported the use of diclofenac. The most common reasons for NSAID use were: headache, back pain and pain located in the extremities (Tab. 2). The average intensity of pain prompting our respondents to take a painkiller was $6.03 \pm 1.99$ on a 10 -point scale, where 0 is no pain and 10 represents the worst pain imaginable.

Overall, on the scale from 1 to 10 , where 1 is the safest medication on the market and 10 the most dangerous, respondents perceived the risk of NSAIDs as $4.27 \pm 2.46$. Risk perception was in line with our prior results from Greece $(4.36 \pm 2.41, \mathrm{p}=0.360)$. On the other hand, our respondents perceived NSAIDs as significantly more dangerous than patients in Slovakia $(3.8 \pm 1.9, \mathrm{p}=0.038)$.

Nearly half of the respondents $(n=79,42.7 \%)$ were familiar with at least some of the potential ADRs of NSAIDs and $21(11.4 \%)$ reported they suffered from ADRs of painkillers in the past. 92 (49.7\%) patients mentioned they never tried to find out more about potential adverse effects, which is a significantly larger percentage than in Slovakia $(27.9 \%, \mathrm{p}=<0.001)$ or Greece $(16.9 \%, \mathrm{p} \leq 0.001)$. In the remainder of the study group, the most commonly utilized sources of information were the printed documentation enclosed with the medication, and their doctor or pharmacist (Tab. 3). Upon further analysis, we discovered that although 107 respondents $(57.8 \%)$ were informed about the possible risks of NSAID treatment by either their doctor, pharmacist, or both, from these only 61 (33.0\% of the entire study population) were informed spontaneously, without the patient actively asking about the possible ADRs (Tab. 4). Healthcare providers in South Dakota were informing their patients spontaneously significantly more often than providers in Slovakia $(15.9 \%, \mathrm{p} \leq 0.001)$, and on par with Greek providers $(36.3 \%, \mathrm{p}=0.631)$.

The most widely known ADRs according to our respondents were liver damage ( $\mathrm{n}=29,15.7 \%$ ), impairment of kidney function $(\mathrm{n}=28,15.1 \%)$ and gastrointestinal problems $(\mathrm{n}=23,12.4 \%)$. Only 2 respondents $(1.1 \%)$ were aware of potential cardiovascular ADRs.

With regards to factors potentially increasing the risk of cardiovascular ADRs of NSAIDs, systemic arterial hypertension $(\mathrm{HTN})$ was the most prevalent $(\mathrm{n}=$ $106,57.3 \%$ ), followed by diabetes mellitus (DM), kidney disease, coronary artery disease (CAD) and CHF (Tab. 5). All the 
Tab. 5. Prevalence of risk factors for cardiovascular adverse effects.

\begin{tabular}{lcc}
\hline Risk factor & Number of patients & Percentage \\
\hline Hypertension & 106 & $57.3 \%$ \\
Diabetes & 61 & $33.0 \%$ \\
Kidney disease & 43 & $23.2 \%$ \\
History of myocardial infarction & 34 & $18.4 \%$ \\
Coronary artery disease & 30 & $16.2 \%$ \\
Congestive heart failure & 29 & $15.7 \%$ \\
Stroke & 24 & $13.0 \%$ \\
Disease of native arteries other than the coronaries & 20 & $10.8 \%$ \\
Deep venous thrombosis & 19 & $10.3 \%$ \\
Pulmonary embolism & 15 & $8.1 \%$ \\
None of the above & 36 & $19.5 \%$ \\
\hline
\end{tabular}

Tab. 6. Use of antithrombotic medications by study participants.

\begin{tabular}{lcc}
\hline Antithrombotic & Number of patients & Percentage \\
\hline Low-dose aspirin & 60 & $32.4 \%$ \\
Warfarin & 19 & $10.3 \%$ \\
Clopidogrel & 17 & $9.2 \%$ \\
Rivaroxaban & 11 & $5.9 \%$ \\
Apixaban & 8 & $4.3 \%$ \\
Dabigatran & 2 & $1.1 \%$ \\
Ticagrelor & 2 & $1.1 \%$ \\
Yes, but unsure of name & 1 & $0.5 \%$ \\
None & 88 & $47.6 \%$ \\
\hline
\end{tabular}

patients on medical treatment for HTN $(105,56.8 \%)$ reported that their blood pressure was well controlled. Over $60 \%$ of patients reported a regular use of an antithrombotic medication, most commonly low dose acetylsalicylic acid (aspirin) (Tab. 6). History of peptic ulcer or upper gastrointestinal bleeding was present in 44 respondents $(23.8 \%)$.

\section{Discussion}

The relatively high average age of our respondents $(63.6 \pm 17.5$ years) can be explained by the inclusion of only patients hospitalized with IM diagnosis and is in line with prior data gathered using a similar methodology in Slovakia (60.9 \pm 13.8$)$ and Greece (57.1 \pm 15.5 years $)(13,14)$. This age group represents the typical population with a high frequency of NSAID use due to a high prevalence of chronic musculoskeletal and connective tissue diseases leading to chronic pain (2). Consequently, it is not surprising that nearly all of our respondents had at least some experience with painkillers, and nearly half of them used NSAIDs on a weekly to monthly basis. The two most widely used analgesics were ibuprofen and paracetamol (acetaminophen in the US), what is in line with our prior data from Slovakia and Greece. Likely, due to country-specific differences in availability, the third most widely used NSAID in South Dakota was naproxen, while in Europe, diclofenac was preferred. From a CV safety standpoint, the US practice appears preferable, since traditionally naproxen has been considered the safest NSAID for patients with heart disease, while diclofenac seemed to be linked to an increase in CV event rates (2).

The perceived risk of NSAIDs was in line with our results from Greece $(4.36 \pm 2.41)$, but appeared higher than the risk perception in Slovakia (3.8 \pm 1.9$)$, or in the cohort from Ireland (2.1, confidence interval 0.7-4.9) $(13,14,15)$. In the light of prior data, it was not surprising to find that over half of the cohort had no knowledge of possible ADRs, which echoed our prior findings in the European cohorts and also was hand in hand with the fact that only 50.3 $\%$ of respondents were trying to obtain information about possible risks of treatment. Interestingly, our respondents showed a significantly less interest in researching possible ADRs than respondents from Slovakia or Greece, yet the number of respondents aware of any ADRs was similar in all 3 cohorts.

With regards to healthcare providers informing their patients about the risk of NSAIDs, we found that significantly more doctors/pharmacists inform their patients in South Dakota and Greece than in Slovakia. There was no statistically significant difference between our cohort and Greece $(p=0.168)$. In cases of patients, who were informed by their provider about the possible risks, we found that a little over half were informed spontaneously, without a direct question from the patient. Overall, 61 (33.0\%) respondents were educated spontaneously, which is in line with our results from Greece (36.3\%), however is significantly more than in Slovakia (15.9\%). The reasons for Slovak providers lagging behind in informing their patients about risks of NSAIDs cannot be determined by the methodology of our study. We can theorize about possible differences in the average length of patient/healthcare provider interaction, differences in the culture of practicing medicine or insufficient emphasis on risk prevention during medical school education in Slovakia being possible contributing factors. Of note, the fact that NSAID risk perception was significantly lower in Slovakia than in Greece or South Dakota indicates that the extra time spent with patient education might in fact make a measurable impact, and thus the time is well spent.

Overall, it seems that in South Dakota, just like in Slovakia and Greece, a proactive approach from the patients is usually needed to gain sufficient knowledge about ADRs of NSAIDs. Of note, only $2(1.1 \%)$ respondents were aware of any potential cardiovascular ADRs, which means that from 107 cases of healthcare providers informing their patients about the risks of treatment, $\mathrm{CV}$ ADRs were not covered in 105 . We can speculate that the awareness and/or risk perception of doctors and pharmacists about CV adverse effects is insufficient, far below the level of awareness of GI complications and kidney damage.

The insufficient knowledge about the potential risks of treatment is alarming, especially since we found that a significant portion of our cohort had risk factors for NSAID mediated ADRs. The most common risk factor was unsurprisingly HTN, which appeared to be well controlled in every patient we interviewed. Interestingly, none of the patients were aware that NSAIDs have the potential to elevate blood pressure and destabilize previously well controlled HTN. It is disconcerting that despite over $60 \%$ of our respondents being on an antithrombotic regimen and one fourth having a history of GI ulcer or bleed, only 9 of them (4.9\%) knew about the increased bleeding risk during NSAID use. 


\section{$771-774$}

\section{Conclusion}

We demonstrated a dangerously low public awareness about NSAID related adverse effects. Even though half of our study group was aware that analgesic treatment might have some unintended consequences, knowledge about the most important, potentially fatal complications was severely lacking. In particular, the awareness of potential adverse CV consequences of NSAID administration was minimal.

Despite a significant percentage of respondents using NSAIDs regularly, while having factors predisposing to adverse effects of NSAIDs, only a third of healthcare providers informed their patients about the possible risks without being directly asked. This puts the initiative into the hands of the patient, who, if not sufficiently proactive, will not have the necessary knowledge about ADRs to allow quick recognition, expeditious discontinuation of treatment and seeking of medical attention.

\section{Learning points}

1. Adverse effects of non-steroidal anti-inflammatory drugs represent a public health problem.

2. Only $12.4 \%$ of patients were familiar with gastrointestinal adverse effects and only $1.1 \%$ were aware of cardiovascular risk.

3. Only one third of providers inform the patients about possible risks spontaneously, without a direct question from the patient.

\section{References}

1. McGettigan P, Henry D. Use of non-steroidal anti-inflammatory drugs that elevate cardiovascular risk: an examination of sales and essential medicines lists in low-, middle-, and high-income countries. PLoS Med 2013; 10: e1001388.

2. Varga Z, Sabzwari SAR, Vargova V. Cardiovascular risk of non-steroidal anti-inflammatory drugs: an under-recognized public health issue. Cureus 2017; 9 (4): e1144.

3. Douthwaite AH: Recent advances in medical diagnosis and treatment. Brit Med J 1938; 1: 1143-1146.
4. Gislason GH, Jacobsen S, Rasmussen JN et al. Risk of death or reinfarction associated with the use of selective cyclooxygenase-2 inhibitors and nonselective non-steroidal antiinflammatory drugs after acute myocardial infarction. Circulation 2006; 113 (25): 2906-2913.

5. Gislason GH. NSAIDs and Cardiovascular Risk. Am Fam Physician 2009; 80 (12): 1366-1368.

6. Lafrance JP, Miller DR. Selective and non-selective non-steroidal antiinflammatory drugs and the risk of acute kidney injury. Pharmacoepidemiol Drug Saf 2009; 18: 923-931.

7. Pan Y, Zhang L, Wang F, Li X, Wang H. Status of non-steroidal antiinflammatory drugs use and its association with chronic kidney disease: A cross sectional survey in China. Nephrology (Carlton) 2014; 19 (10): 655-660.

8. Olsen AM, Fosbøl EL, Lindhardsen $\mathbf{J}$ et al. Long-Term Cardiovascular Risk of NSAID Use According to Time Passed After First-Time Myocardial Infarction: A Nationwide Cohort Study. Circulation 2012; 126 (16): 1955-1963.

9. Park K, Bavry AA. Risk of stroke associated with nonsteroidal antiinflammatory drugs. Vasc Health Risk Manag 2014; 10: 25-32.

10. Armstrong EP, Malone DC. The impact of nonsteroidal anti-inflammatory drugs on blood pressure, with an emphasis on newer agents. Clin Ther 2003; 25: 1-18.

11. Solomon DH, Schneeweiss S, Levin R, Avorn J. Relationship between COX-2 specific inhibitors and hypertension. Hypertension 2004; 44: $140-145$.

12. Baxter K (Ed). Stockley's drug interactions (ninth edition). London; Pharmaceutical Press, 2010: 1792 p.

13. Varga Z, Kriska M, Kristova V, Petrova M. Analysis of non-steroidal anti-inflammatory drug use in hospitalized patients and perception of their risk. Interdiscip Toxicol 2013; 6 (3): 141-144.

14. Karakitsiou M, Varga Z, Kriska M, Kristova V. Non-steroidal anti-inflammatory drugs: perception of their risk in Greece. Bratisl Med J 2017; 118 (7): 427-430.

15. Cullen G, Kelly E, Murray FE. Patients' knowledge of adverse reactions to current medications. Br J Clin Pharmacol 2006; 62 (2): 232-236.

Received May 26, 2020. Accepted August 18, 2020. 